¡div class=" moz-text-flowed" style=" font-family: -moz-fixed" i

\title{
ON TWO CONJECTURES CONCERNING CONVEX CURVES
}

\author{
V. SEdykh And B. Shapiro \\ Department of Mathematics, University of Oil and Gas (Gubkin) \\ Moscow 117036, Russia, sedykh@mccme.ru \\ Department of Mathematics, University of Stockholm \\ S-10691, Sweden, shapiro@math.su.se
}

\begin{abstract}
In this paper we recall two basic conjectures on the developables of convex projective curves, prove one of them and disprove the other in the first nontrivial case of curves in $\mathbb{R} P^{3}$. Namely, we show that i) the tangent developable of any convex curve in $\mathbb{R} P^{3}$ has degree 4 and ii) construct an example of 4 tangent lines to a convex curve in $\mathbb{R} P^{3}$ such that no real line intersects all four of them. The question (discussed in [EG1] and [So4]) whether the second conjecture is true in the special case of rational normal curves still remains open.
\end{abstract}

\section{$\S 1$. INTRODUCTION AND RESULTS}

We start with some important notions.

Main Definition. A smooth closed curve $\gamma: S^{1} \rightarrow \mathbb{R} P^{n}$ is called locally convex if the local multiplicity of intersection of $\gamma$ with any hyperplane $H \subset \mathbb{R} P^{n}$ at any of the intersection points does not exceed $n=\operatorname{dim} \mathbb{R} P^{n}$ and globally convex or just convex if the above condition holds for the global multiplicity, i.e for the sum of local multiplicities.

Local convexity of $\gamma$ is a simple requirement of nondegeneracy of the osculating Frenet $n$-frame of $\gamma$, i.e. of the linear independence of $\gamma^{\prime}(t), \ldots, \gamma^{(n)}(t)$ for any $t \in S^{1}$. Global convexity is a nontrivial property equivalent to the fact that the $(n+1)$-tuple of $\gamma$ 's homogeneous coordinates forms a Tschebychev system of functions, see e.g. [KS]. The simplest examples of convex curves are the rational normal curve $\rho_{n}: t \mapsto\left(t, t^{2}, \ldots, t^{n}\right)$ (in some affine coordinates on $\mathbb{R} P^{n}$ ) and the standard trigonometric curve $\tau_{2 k}: t \mapsto$ $\left(\sin t, \cos t, \sin 2 t, \cos 2 t, \ldots, \sin 2 k t, \cos 2 k t\right.$ ) (in some affine coordinates on $\mathbb{R} P^{2 k}$ ).

Definition. The $k$-th developable $D_{k}(\gamma)$ of a curve $\gamma: S^{1} \rightarrow \mathbb{R} P^{n}$ is the union of all $k$-dimensional osculating subspaces to $\gamma$. The hypersurface $D(\gamma)=D_{n-2}(\gamma)$ is called the developable hypersurface of $\gamma$. $(D(\gamma)$ was called the discriminant of $\gamma$ in [SS].)

Note that $D_{k}(\gamma)$ can be considered as the image of the natural associated map $\gamma_{k}$ : $S^{1} \times \mathbb{R} P^{k} \rightarrow \mathbb{R} P^{n}$.

Definition. Let $M$ be a compact manifold of some dimension $l \leq n$ and $\phi: M \rightarrow \mathbb{R} P^{n}$ be a smooth map. By the degree of $\phi(M)$ we understand the supremum of the number of its intersection points with generic $(n-l)$-dilmensional subspaces. Recall that an $(n-l)$ dimensional subspace $L$ is called generic w.r.t $\phi(M)$ if the intersection $L \cap \phi(M)$ consists 
subspaces for $\phi(M)$ (for example, if $\operatorname{dim} \phi(M)<\operatorname{dim}(M)$ ) then we set the degree of $\phi(M)$ equal to zero. (It is rather obvious that if the Jacobian of $\phi$ is nondegenerate at least at one point of $M$ then generic $(n-l)$-dimensional subspaces exist.)

In particular, one can consider the degree of $D_{k}(\gamma)$ which is positive unless $\operatorname{dim} D_{k}(\gamma)<$ $k+1$.

REMARK. By definition $\gamma$ is convex if and only if the degree of $\gamma=D_{0}(\gamma)$ (considered as the image of the map $\gamma: S^{1} \rightarrow \mathbb{R} P^{n}$ ) equals $n$. It is well-known that $\gamma$ is convex if and only if the dual curve $\gamma^{*} \in\left(\mathbb{R} P^{n}\right)^{*}$ is convex, see e.g. [A1]. Thus $\gamma$ is convex if and only if the degree of $D_{n-1}(\gamma)$ as the image of associated map $\gamma_{n-1}: S^{1} \times \mathbb{R} P^{n-1} \rightarrow \mathbb{R} P^{n}$ equals $n$. The following conjecture generalizes the latter fact.

Conjecture on the $k$-th developable. A curve $\gamma: S^{1} \rightarrow \mathbb{R} P^{n}$ is convex if and only if for some (and then for all) $k=0, \ldots, n-1$ the degree of its $k$-th developable $D_{k}(\gamma)$ equals $(k+1)(n-k)$.

(Note that $(k+1)(n-k)$ is the dimension of the Grassmannian of projective $k$ dimensional subspaces in $P^{n}$.)

As a special case one gets

Conjecture on the developable hypersurface. A curve $\gamma: S^{1} \rightarrow \mathbb{R} P^{n}$ is convex if and only if the degree of its developable hypersurface $D(\gamma) \subset \mathbb{R} P^{n}$ equals $2 n-2$.

REMARK. The conjecture on the $k$-th developable can be verified by a straightforward degree count in the case when $\gamma$ is the rational normal curve. Our first result is the following

Theorem A. A curve $\gamma: S^{1} \rightarrow \mathbb{R} P^{3}$ is convex if and only if the degree of its developable hypersurface $D(\gamma)$ equals 4 .

In order to formulate the second conjecture recall that for a generic $(k+1)(n-k)$-tuple of $k$-dimensional complex subspaces in $\mathbb{C} P^{n}$ there exist $\sharp_{k, n}=\frac{1 ! 2 ! \ldots(n-k-1) !((k+1)(n-k)) !}{(k+1) !(k+2) ! \ldots .(n) !}$ projective complex subspaces of dimension $(n-k-1)$ in $\mathbb{C} P^{n}$ intersecting each of the above $k$-dimensional susbspaces. This is a classical result due to H. Schubert, see [Sch]. (The number $\sharp_{k, n}$ is the degree of the Grassmanian of projective $k$-dimensional subspaces in $P^{n}$ considered as a projective variety embedded using Plücker coordinates.)

Conjecture on total reality. For the real rational normal curve $\rho_{n}: S^{1} \rightarrow \mathbb{R} P^{n}$ and any $(k+1)(n-k)$-tuple of pairwise distinct real projective $k$-dimensional osculating subspaces to $\rho_{n}$ there exist $\sharp_{k, n}$ real projective subspaces of dimension $(n-k-1)$ in $\mathbb{R} P^{n}$ intersecting each of the above osculating subspaces.

The latter conjecture is sometimes referred to as the B.\&M.Shapiro conjecture and has the following appealing interpretation in terms of real algebraic geometry. Consider a generic degree $n$ rational curve: $\mu: \mathbb{C} P^{1} \rightarrow \mathbb{C} P^{k}$. Such a curve has exactly $(k+1)(n-k)$ inflection points. (An inflection point of a space curve $\mu$ is a point where the osculating Frenet frame $\left(\mu^{\prime}, \mu^{\prime \prime}, \ldots, \mu^{(k)}\right)$ is degenerate.)

Conjecture on total reality restated. In the above notation assume additionally that the inverse images of all inflection points lie on $\mathbb{R} P^{1} \subset \mathbb{C} P^{1}$. Then $\mu$ is a real 
rational curve up to a projective transformation in $\mathbb{C} P^{k}$, i.e. there exists a projective transformation of $\mathbb{C} P^{k}$ making $\mu$ into a curve invariant under the complex conjugations in the preimage and the image.

The conjecture on total reality in the case $k=1$, i.e. for rational functions in one variable was settled in [EG1]. Extensive numerical support for its validity for $k>1$ can be found in [So4] and its intriguing generalization to the case of manifolds of (in)complete flags is contained in [RSSS].

Earlier the second author proposed a generalized conjecture on total reality saying that the above conjecture should be valid for all convex curves in $\mathbb{R} P^{n}$ (and not just for the rational normal curve), see [So4]. But this generalized conjecture on total reality fails as shown by the next result.

Theorem B. There exists a convex curve $\gamma: S^{1} \rightarrow \mathbb{R} P^{3}$ and a 4-tuple of its tangent lines with no real lines in $\mathbb{R} P^{3}$ intersecting all four tangents.

The most delicate open problem related to the present paper is to find a natural subset of the set of convex curves for which the conjecture of total reality might hold.

REMARKS. One of the implications in Theorem A was earlier proven in [SS]. The conjecture on total reality was discussed in a number of papers, see [EG1], [So1]-[So4]. The structure of the paper is as follows. $§ 2-3$ contain the proofs of Theorems A and B resp. $\S 4$ discusses the above conjectures in terms of Schubert calculus. In particular, it contains a stronger version of the total reality conjecture closely related to the transversality conjecture due to F. Sottile.

ACKnowledgements. The first author is sincerely grateful to the Department of Mathematics, University of Stockholm for the hospitality and to the Department of Mathematics, Royal Institute of Technology for the financial support of his visit to Sweden in April 2002 when this project was started. The second author wants to emphasize the importance of his contacts with A. Gabrielov, A. Eremenko, M. Shapiro and, especially, with F. Sottile.

\section{$\S 2$ Proof of Theorem A}

The idea of the proof of Theorem $\mathrm{A}$ is as follows. We will show that the degree of the developable hypersurface is either identically equal to 4 or strictly greater than 4 for all curves within any connected component of the space of locally convex curves in $\mathbb{R} P^{3}$. (The main technical tool is Proposition 1 below.) After that in order to settle Theorem A one just has to calculate the degree of the developable hypersurface for some suitable representative in each such connected component, see Proposition 2.

Take a smooth 1-parameter family of smooth closed curves $\gamma_{t}: S^{1} \rightarrow \mathbb{R} P^{3}, t \in[0,1]$ and assume that:

(*) for any $t \in(0,1]$ the curve $\gamma_{t}$ is locally convex and the degree of its developable hypersurface $D\left(\gamma_{t}\right)$ equals 4 ;

$(\star \star)$ the degree of the developable hypersurface $D_{0}=D\left(\gamma_{0}\right)$ exceeds 4 .

The next technical statement is the key step in the proof of Theorem A. 
Proposition 1. Under the above assumptions the curve $\gamma_{0}$ is not locally convex, i.e. has inflections.

To prove this result we assume that $\gamma_{0}$ is locally convex and get a contradiction with ( $\star$ ) by finding a line intersecting $D_{0}$ transversally more than 4 times.

First of all note that any curve $\tilde{\gamma}$ sufficiently close to $\gamma_{0}$ in $C^{1}$-topology is locally convex. Hence, every irreducible component of a germ of the developable hypersurface of $\tilde{\gamma}$ at any point is either a smooth germ or is diffeomorphic to a germ of a half-cubical cuspidal edge with singularities at $\tilde{\gamma}$. The number of local irreducible components of a germ of the developable hypersurface at any point is finite. Moreover, we can assume that that the family $\gamma_{t}$ is generic in the sense that the front of tangent planes (i.e. the developable hypersurface of the dual curve) to the curve $\gamma_{t}$ has only generic perestroikas at the moment $t=0$. These perestroikas were listed in [A2], p.2.

The next lemma collects restrictions valid for a locally convex $\gamma_{0}$ satisfying $(\star)$ and $(\star \star)$ in the increasing order of strength. (Recall that our aim is to prove that such a $\gamma_{0}$ cannot exist.)

2.1. LEMMA. If $\gamma_{0}$ is locally convex and satisfies ( $\star$ ) and ( $\left.\star \star\right)$ then

1) $\gamma_{0}$ cannot intersect any of its osculating planes transversally;

2) $\gamma_{0}$ has no self-intersections;

3) $\gamma_{0}$ has exactly one common point with any of its tangent lines;

4) $\gamma_{0}$ has exactly one common point with any of its osculating planes.

Obviously, 4) $\Rightarrow 3$ ) $\Rightarrow 2$ ) $\Rightarrow 1$ ).

Proof. To prove 1) notice that if $\gamma_{0}$ transversally intersects the plane osculating it at a point $p$ at some point $q \neq p$, then for any sufficiently small $t$ the plane osculating the curve $\gamma_{t}$ at some point $\tilde{p}$ close to $p$ transversally intersects $\gamma_{t}$ at some point $\tilde{q}$ close to $q$. By continuity the point $\gamma_{t}(\tilde{q})$ does not lie on the tangent line to $\gamma_{t}$ at $\tilde{p}$. The line passing through the points $\gamma_{t}(\tilde{p}), \gamma_{t}(\tilde{q})$ intersects $D\left(\gamma_{t}\right)$ with the multiplicity $>4$ contradicting $(\star)$.

To prove 2) notice that the osculating plane to one of the local irreducible components of the curve $\gamma_{0}$ at its self-intersection point intersects another irreducible component transversally (since our family $\gamma_{t}$ is chosen generic by assumption). But this is impossible by the above item 1).

To prove 3) assume that the tangent line to $\gamma_{0}$ at a point $p$ intersects $\gamma_{0}$ at some point $q \neq p$. Then $\gamma_{0}(p) \neq \gamma_{0}(q)$ by item 2) and the plane osculating $\gamma_{0}$ at $p$ is tangent to $\gamma_{0}$ at $q$ by item 1). The plane osculating $\gamma_{0}$ at $q$ is transversal to the line passing through the points $\gamma_{0}(p)$ and $\gamma_{0}(q)$ (since the family $\gamma_{t}$ is generic). Hence for any sufficiently small $t$ there exists a point $\tilde{p}$ close to $p$ and a line $l$ in $\mathbb{R} P^{3}$ passing through $\gamma_{t}(\tilde{p})$ such that: a) $l$ belongs to the plane osculating $\gamma_{t}$ at $\tilde{p}$; b) $l$ is not tangent to $\gamma_{t}$ at $\tilde{p}$; c) $l$ intersects the irreducible component of the surface $D\left(\gamma_{t}\right)$ at the point $\gamma_{t}(\tilde{q})$ at two smooth points. The total multiplicity of the intersection $l \cap D\left(\gamma_{t}\right)$ exceeds 4 contradicting $(\star)$.

To prove 4) consider the plane $\pi$ osculating $\gamma_{0}$ at a point $p$. Assume that $\gamma_{0}$ intersects $\pi$ at some point $q \neq p$. Then $\pi$ is tangent to $\gamma_{0}$ at $q$ (by item 1$)$ ), $\gamma_{0}(p) \neq \gamma_{0}(q)$ (by item 2 )) and the line $l$ passing through the points $\gamma_{0}(p), \gamma_{0}(q)$ is not tangent to $\gamma_{0}$ at these points (by item 3)). There are two possible cases to consider: 
i) the plane osculating $\gamma_{0}$ at the point $q$ is transversal to the line $l$. In this case for any sufficiently small $t$ the plane osculating the curve $\gamma_{t}$ at some point $\tilde{p}$ close to $p$ is tangent to $\gamma_{t}$ at some point $\tilde{q}$ close to $q$. It is clear, that the line passing through the points $\gamma_{t}(\tilde{p}), \gamma_{t}(\tilde{q})$ is not tangent to $\gamma_{t}$ at these points and intersects $D\left(\gamma_{t}\right)$ with the multiplicity $>4$.

ii) the plane $\pi$ osculates $\gamma_{0}$ at $q$. Hence, for any sufficiently small $t$ the plane osculating $\gamma_{t}$ at any point $\tilde{p}$ close to $p$ intersects $\gamma_{t}$ at some point $\tilde{q}$ close to $q$. As before the line passing through the points $\gamma_{t}(\tilde{p}), \gamma_{t}(\tilde{q})$ is not tangent to $\gamma_{t}$ at these points and intersects $D\left(\gamma_{t}\right)$ with multiplicity $>4$.

Thus we get a contradiction with $(\star)$ in both cases.

As above denote by $L$ a line in $\mathbb{R} P^{3}$ which is not tangent to $\gamma_{0}$ and intersects $D_{0}$ with total multiplicity $>4$. The next lemma describes restrictions on $L$ in the case when $\gamma_{0}$ is locally convex and satisfies $(\star)$ and $(\star \star)$.

2.2. LEMMA. Under the above assumptions the line $L$ must be tangent to every smooth irreducible component of the surface $D_{0}$ at each intersection point of $L \cap D_{0}$.

Proof. We will consequently exclude the possibility of having a given number of transversal intersections. Namely, the line $L$ cannot intersect $D_{0}$ transversally at more than 4 smooth points since otherwise $L$ will intersect $D\left(\gamma_{t}\right)$ at least 5 times for all sufficiently small $t$.

Let us show that $L$ cannot intersect $D_{0}$ transversally at 3 or 4 smooth points. Indeed, in this case $L$ intersects $D_{0}$ at some point $P$ with the local multiplicity greater than 1 . If $P$ belongs to $\gamma_{0}$ then for any small $t$ any line close to $L$ and intersecting the curve $\gamma_{t}$ at a point close to $P$ will intersect $D\left(\gamma_{t}\right)$ with multiplicity $>4$. If $L$ is tangent to a smooth local irreducible component of the surface $D_{0}$ at the point $P$ then again for any small $t$ any line close to $L$ and tangent to a smooth local irreducible component of the surface $D\left(\gamma_{t}\right)$ at a point near $P$ will intersect $D\left(\gamma_{t}\right)$ with multiplicity $>4$.

Finally, suppose that $L$ intersects $D_{0}$ transversally at 1 or 2 smooth points. Then $L$ intersects two local irreducible components $D_{0}^{1}, D_{0}^{2}$ of the surface $D_{0}$ at some points $P_{1}, P_{2}$ with the local multiplicities at least 2. By Lemma 2.1 items 2) and 4), there are two cases to consider:

a) $P_{1}, P_{2}$ belong to the curve $\gamma_{0}$ and $P_{1} \neq P_{2}$. In this case for any small $t$ the line passing through any points $\tilde{P}_{1}, \tilde{P}_{2}$ of the curve $\gamma_{t}$ close to $P_{1}, P_{2}$ resp. intersects $D\left(\gamma_{t}\right)$ with multiplicity $>4$.

b) $P_{1}, P_{2}$ do not lie on the curve $\gamma_{0}$. In this case the line $L$ is the intersection of the tangent planes to smooth components $D_{0}^{1}, D_{0}^{2}$ at the points $P_{1}, P_{2}$ (these planes are transversal). Hence, for any small $t$ there exists a line in $\mathbb{R} P^{3}$ close to $L$ and tangent to two smooth irreducible components of the surface $D\left(\gamma_{t}\right)$ at the points near $P_{1}, P_{2}$. This line intersects $D\left(\gamma_{t}\right)$ with the multiplicity $>4$.

Thus, $L$ cannot intersect $D_{0}$ transversally at its smooth points.

Lemmas 2.1 and 2.2 leave us with only two possible (and dual to each other) positions of the line $L$ w.r.t $\gamma_{0}$ :

(i) the line $L$ intersects $\gamma_{0}$ at least at 3 different points and does not lie in osculating planes to $\gamma_{0}$; 
(ii) the line $L$ lies in at least 3 different osculating planes to the curve $\gamma_{0}$ and does not intersect $\gamma_{0}$.

The next lemma rules out these remaining possibilities and accomplishes the proof of Proposition 1.

2.3. Lemma. In both cases (i) and (ii) one can find a line close to $L$ which intersects $D_{0}$ transversally at more than 4 points.

Proof. Indeed, consider the central projection $\varrho: \mathbb{R} P^{3} \rightarrow \mathbb{R} P^{2}$ from some point $O \in L$. (In each of the cases we will choose the point $O$ differently.)

(i) Assume that the point $O$ does not belong to $\gamma_{0}$. Then $\varrho\left(\gamma_{0}\right)$ has at least 3 irreducible locally convex components $c_{1}, c_{2}, c_{3}$ at the point $\varrho(L)$. Every curve $c_{i}, i=1,2,3$ separates a small neighborhood of this point into two open domains one of which (denoted by $U_{i}$ ) satisfies the property that each point of $U_{i}$ belongs to two lines tangent to the curve $c_{i}$. The intersection $U=U_{1} \cap U_{2} \cap U_{3}$ is nonempty. (See Fig.1a below illustrating the most delicate case.) Then for any point $P \in U$ the line $\varrho^{-1}(P)$ intersects at least 6 local irreducible components of $D_{0}$ transversally.

(ii) Let $L$ belong to the planes osculating $\gamma_{0}$ at the points $P_{1}, P_{2}, P_{3}$. Then the tangent lines to $\gamma_{0}$ at these points cannot intersect the line $L$ at the same point since the family $\gamma_{t}$ is taken generic. Take the point $O \in L$ which belongs only to one of the tangent lines to $\gamma_{0}$ at the points $P_{1}, P_{2}, P_{3}$. Assume, for example, that $O$ belongs to the line tangent to $\gamma_{0}$ at $P_{1}$. Then the projection $c_{i}$ of a germ of $\gamma_{0}$ at the point $P_{i}$ has a half-cubical cusp at the point $\varrho\left(P_{i}\right)$ for $i=1$ and cubical inflections for $i=2,3$.

It is easy to see that the projections of the planes osculating $\gamma_{0}$ at the points $P_{1}, P_{2}, P_{3}$ separate a small neighborhood of the point $\varrho(L)$ into six parts one of which (denoted by $U)$ satisfies the property that each point of $U$ belongs to two lines tangent to each curve $c_{i}, i=2,3$ as well as one line tangent to the curve $c_{1}$, see Fig.1b. Then for any point $P \in U$ the line $\varrho^{-1}(P)$ intersects at least 6 irreducible components of the surface $D_{0}$ transversally. Thus both cases (i) and (ii) are impossible. This contradicts our initial assumption about the local convexity of $\gamma_{0}$ and accomplishes the proof of Proposition 1.

Proposition 1 implies that if a connected component of the space of locally convex curves contains a curve such that the degree of its developable hypersurface is 4 , then the degree of the developable hypersurface of every curve in this component is 4 . In the (unique up to the choice of orientation) component containing convex curves the degree of the developable hypersurfaces equals 4 since this fact holds for the rational normal curve. Hence Theorem A follows from the next statement. 

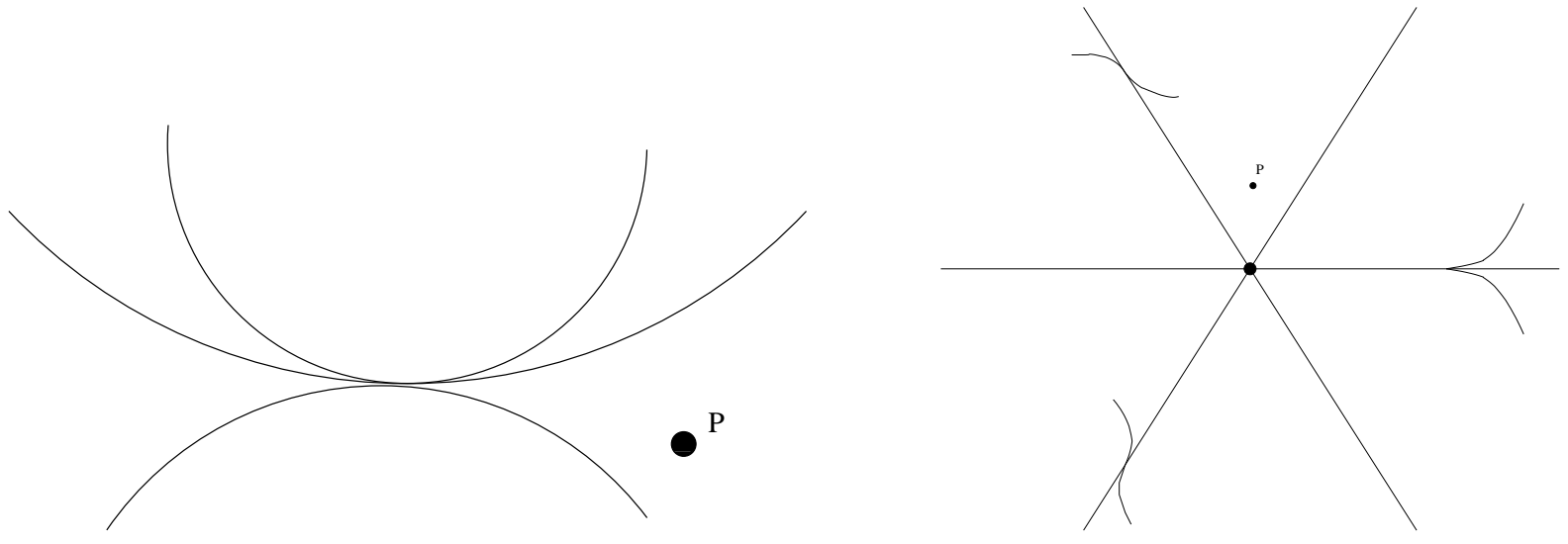

Fig. 1. Illustrations to Lemma 2.3(I) AND 2.3(II).

Proposition 2. Every connected component of the space of locally convex curves different from two components consisting of convex curves only contains a curve with the degree of its developable hypersurface $>4$.

Proof. By [MSh] there exist 5 connected components of the space of locally convex right projective curves in $\mathbb{R} P^{3}$. (The word 'right' means that the Frenet frames of all these curves represent just one fixed 'right' orientation of $\mathbb{R} P^{3}$.) Three of these components (including the component of convex curves) contain noncontractible curves and the other two contain contractible curves. The invariant distinguishing components is the mod 2 element of $\pi_{1}\left(\mathrm{SO}_{4}\right)$.

Moreover, if $\xi$ is a contractible locally convex curve whose lift to $\mathrm{SO}_{4}$ realizes a nontrivial element of $\pi_{1}\left(\mathrm{SO}_{4}\right)$ then we can choose the following representatives of all five connected components. Namely, $\rho, \rho+\xi$ and $\rho+\xi^{2}$ represent three components of noncontractible curves; $\xi$ and $\xi^{2}$ represent two components of contractible curves. Here $\rho$ is the rational normal curve in $\mathbb{R} P^{3}$, the ${ }^{\prime}+^{\prime}$-sign means that we append the second curve to the endpoint of the first curve with the same tangent direction. Finally, the expression $\xi^{2}$ denotes the curve $\xi$ traversed twice. (The lift of a locally convex right curve to $\mathrm{SO}_{4}$ is obtained as follows, see also $\S 3$. A right curve in $\mathbb{R} P^{3}$ first lifts canonically to the sphere $S^{3} \subset \mathbb{R}^{4}$ and then its extended Frenet frame lifts it to $S_{4}$. One can lift all locally convex curves, both right and left to $\mathrm{O}_{4}$.)

Here and below by an extended Frenet frame of a projective curve $\gamma: S^{1} \rightarrow \mathbb{R} P^{3}$ we mean the frame $\left(\tilde{\gamma}, \tilde{\gamma}^{\prime}, \tilde{\gamma}^{\prime \prime}, \tilde{\gamma}^{\prime \prime \prime}\right)$ of the canonical lift $\tilde{\gamma}: S^{1} \rightarrow S^{3} \subset \mathbb{R}^{4}$ of $\gamma$ described above.

Therefore if we can find an example of $\xi$ such that $D(\xi)$ has degree $>4$ Proposition 2 will follow. An example of $\xi$ given in Fig. 2 below was obtained by a suitable modification of fig 2, p. 111 in [To]. (The shown modification was required since the original picture in [To] which was supposed to present the velocity vector of an apropriate $\xi$ must necessarily contain spherical inflection points by the theorem of Fenchel on the existence of inflections on a noncontractible embedded curve on $\left.\mathbb{R} P^{2},[\mathrm{Fe}].\right)$

Let us now show that the developable hypersurface $D(\xi)$ has degree at least 6 . In fact, we show that already the piece $\tilde{\xi}$ of $\xi$ corresponding to the fragment of $\xi^{\prime}$ going around the north pole three times gives a contribution of at least 6 to the degree. Indeed, consider 
the $(x, y)$-plane $\Pi$. The velocity vector of $\tilde{\xi}$ is never vertical. Therefore the projection $\pi \xi$ of $\tilde{\xi}$ onto $\Pi$ along the $z$-axis is smooth, convex and its velocity vector makes three complete turns. Let us choose some convex disc $\mathfrak{D}$ containing $\pi \xi$. We check now that $\Pi \backslash D$ contains a point having at least 6 different tangents to $\pi \xi$. Indeed, the union of all tangents to any segment of a convex curve of $\mathbb{R}^{2}$ whose tangent vector makes a halfturn covers the whole $\mathbb{R}^{2}$ except for the strip between the parallel tangents at the endpoints. Therefore, the segment whose velocity vector makes three complete turns covers $\Pi$ minus some strip at least 6 times. Finally, choose the line $l l$ parallel to the $z$-axis and passing through the chosen point on $\Pi$. We have just shown that the developable hypersurface $D(\xi)$ of $\xi$ crosses $l l$ at least 6 times.

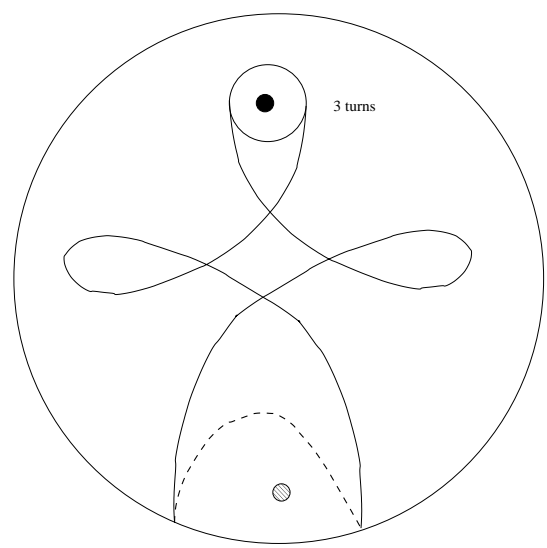

Fig. 2. Velocity Vector of An APPROPRiate RePresentative $\xi$.

Explanations to Fig. 2. This picture represents the graph of the velocity vector $\xi^{\prime}$ of $\xi$ parameterized by the arclength. Therefore $\left|\xi^{\prime}\right|=1$ implying that $\xi^{\prime}$ lies on $S^{2} \subset \mathbb{R}^{3}$. (The black dots on the picture stand for the north resp. south poles.) The existence of a closed $\xi$ with a given $\xi^{\prime}$ follows from a result of Fenchel's claiming that a closed nonparameterized curve in $\mathbb{R}^{n}$ is realizable (with a suitable parametrization) as the velocity vector of another closed curve if and only if its convex hull contains the origin. The curve $\xi$ is contractible in $\mathbb{R} P^{3}$ (since it lies in $\mathbb{R}^{3}$ ) and its lift to $S_{3}$ by the usual Frenet frame realizes the nontrivial element in $\pi_{1}\left(S O_{3}\right)$. This last fact follows from the following observation. We can deform $\xi^{\prime}$ in one hemisphere and the resulting curve makes an odd number of turns in this hemisphere. This implies using the triviality of the fibration $S_{4} \rightarrow S^{3}$ restricted to a hemisphere that the lift of $\xi$ to $\mathrm{SO}_{4}$ by the extended Frenet frame realizes a nontrivial element in $\pi_{1}\left(\mathrm{SO}_{4}\right)$ as well.

\section{§3. Proof of Theorem B.}

In order to prove theorem $\mathrm{B}$ we first recall the relation between the osculating flags to a convex curve and totally positive uppertriangular matrices, see [BSh].

Some Notation. Let $F_{n+1}$ denote the space of complete (projective) flags in $\mathbb{R} P^{n}$ or, equivalently, linear flags in $\mathbb{R}^{n+1}$. For a given locally convex curve $\gamma: S^{1} \rightarrow \mathbb{R} P^{n}$ we 
define its flag lift $\gamma_{F}: S^{1} \rightarrow F_{n+1}$ to be the curve of its complete osculating flags. Two flags $f_{1}$ and $f_{2}$ are called transversal if each pairs of subspaces (one from each flag) is transversal. The set $T n_{f}$ of flags nontransversal to a given flag $f$ is called the train or the flag hyperplane associated to $f$. Note that $T n_{f}$ is the union of all Schubert cells of positive codimension of the standard Schubert cell decomposition of $F_{n}$ w.r.t $f$.

One has the following fundamental criterion of convexity going back to G. Pólya, see [Po] and [BSh].

3.1. Theorem. A curve $\gamma: S^{1} \rightarrow \mathbb{R} P^{n}$ is convex if and only if any two distinct osculating flags are transversal.

A slight refinement of the above statement allows us to characterize all configurations of complete flags which can be realized as osculating to some convex curve in appropriate semigroup terms. We will use homogeneous coordinates and oriented complete flags. A complete linear flag in $\mathbb{R}^{n+1}$ is called oriented if any of its subspaces is equipped with an orientation. The set of all complete oriented flags in $\mathbb{R}^{n+1}$ is isomorphic to the group $O_{n+1}$ which covers $F_{n+1}$ with the discrete fiber of cardinality $2^{n+1}$ consisting of all choices of orientations for the subspaces. Notice that any projective curve $\gamma: S^{1} \rightarrow \mathbb{R} P^{n}$ can be canonically lifted to the curve $\tilde{\gamma}$ on the sphere $S^{n} \subset \mathbb{R}^{n+1}$. (If $\gamma$ is contractible then $\tilde{\gamma}$ is closed and if $\gamma$ is noncontractible then $\tilde{\gamma}$ ends at the antipodal points. Note that convex curves in $\mathbb{R} P^{2 n+1}$ are noncontractible while convex curves in $\mathbb{R} P^{2 n}$ are contractible.) Any locally convex $\gamma: S^{1} \rightarrow \mathbb{R} P^{n}$ can therefore be lifted by using its extended Frenet frame in $\mathbb{R}^{n+1}$ to the space $O_{n+1}$ of complete oriented flags. (The lift of a contractible curve is a closed curve on $O_{n+1}$, the lift of a noncontractible is 'antipodal'.) As above we say that two oriented flags $\tilde{f}_{1}$ and $\tilde{f}_{2}$ are transversal if their underlying usual flags $f_{1}$ and $f_{2}$ (obtained by forgetting all orientations) are transversal. The Schubert cell decomposition of $F_{n+1}$ w.r.t. some complete flag lifts to $O_{n+1}$ and the lifted decomposition contains, for example, $2^{n+1}$ open Schubert cells all projecting to the only open Schubert cell in $F_{n+1}$.

Let us fix a basis $\left(e_{1}, \ldots, e_{n+1}\right)$ in $\mathbb{R}^{n+1}$. Let $f_{+}$be the complete linear flag whose $i$-dimensional subspaces are spanned by $e_{1}, \ldots, e_{i}$ and $f_{-}$be its opposite flag whose $i$ dimensional subspaces are spanned by $e_{n+1}, \ldots, e_{n-i+2}$. Analogously, let $\tilde{f}_{+}$be the complete oriented linear flag whose $i$-dimensional subspaces are spanned by $e_{1}, \ldots, e_{i}$ with the orientation given by $e_{1} \wedge \ldots \wedge e_{i}$ and $\tilde{f}_{-}$be its opposite flag, i.e. the flag whose $i$-dimensional subspaces are spanned by $e_{n+1}, \ldots, e_{n-i+2}$ with the orientation given by $e_{n+1} \wedge \ldots \wedge e_{n-i+2}$. The open Schubert cell (affine chart) on $F_{n+1}$ consisting of all flags transversal to $f_{-}$can be identified with the group $T$ of all uppertriangular $(n+1) \times(n+1)$ matrices (taken in the chosen basis $\left.\left(e_{1}, \ldots, e_{n+1}\right)\right)$ whose diagonal entries equal 1. Analogously, the open Schubert cell on $O_{n+1}$ consisting of all oriented flags transversal to $f_{-}$ and containing $\tilde{f}_{+}$can be identified with the group $T$ as well. (As usual, the $i$-dimensional subspace of the flag corresponding to some matrix is spanned by its $i$ first rows and its orientation is induced by the wedge of these rows.)

Definition. A matrix $M \in T$ is called uppertriangular totally positive (resp. totally nonnegative) if any of its minors which does not vanish due to uppertriangularity is positive (resp. any of its minors is nonnegative), see e.g. [Ga].

REMARK. The set $T^{+} \subset T$ of all totally positive matrices is a semigroup and is contractible as a topological space. 
Definition. A sequence $\left\{\tilde{f}_{1}, \tilde{f}_{2}, \ldots, \tilde{f}_{r}\right\}$ of matrices in $T^{+} \subset T$ is called totally positive if each $\tilde{f}_{i+1}$ lies in the cone $\tilde{f}_{i} \circ T^{+}$, i.e. there exists a totally positive matrix $M_{i, i+1}$ such that $\tilde{f}_{i+1}=\tilde{f}_{i} M_{i, i+1}$. If $T^{+}$is identified with is the open cell in $O_{n+1}$ w.r.t. some basis $\left(e_{1}, \ldots, e_{n+1}\right)$ in $\mathbb{R}^{n+1}$ then we say that the sequence of oriented flags $\left\{\tilde{f}_{1}, \tilde{f}_{2}, \ldots, \tilde{f}_{r}\right\}$ is totally positive w.r.t. this basis.

Let us choose an orientation of $S^{1}$ and a reference point $O \in S^{1}$. This choice identifies $S^{1} \backslash O$ with the interval $[0,2 \pi)$.

3.2. TheOrem, SEE [BSh]. For a given convex curve $\gamma: S^{1} \rightarrow \mathbb{R} P^{n}$ and a given sequence of points $\left\{t_{1}<t_{2}<\ldots<t_{r}\right\} \in[0,2 \pi)=S^{1} \backslash O$ there exists a basis in $\mathbb{R}^{n+1}$ such that the sequence $\left\{\tilde{f}_{1}, \ldots, \tilde{f}_{r}\right\}$ of extended osculating flags to $\tilde{\gamma}$ at the points $\left\{t_{1}<t_{2}<\ldots<t_{r}\right\}$ is totally positive w.r.t. the above mentioned basis. Conversely, for any given sequence of totally matrices there exists a convex curve $\gamma: S^{1} \rightarrow \mathbb{R} P^{n}$ and a set of points $\left\{t_{1}<t_{2}<\ldots<t_{r}\right\} \in[0,2 \pi)$ such that this sequence coincides with the sequence of extended osculating oriented flags to this curve at these points w.r.t. an appropriately chosen basis.

Proof. Let us sketch the proof, see details in [BSh]. The lifts of locally convex curves to $O_{n+1}$ obey the following remarkable Cartan distribution of cones on $O_{n+1}$. Let $\tilde{f}=\left(\mathbf{l}_{1} \subset\right.$ $\cdots \subset \mathbf{l}_{n+1}$ ) be a complete oriented flag on $\mathbb{R}^{n+1}$, where $\mathbf{l}_{i}$ denotes its $i$-dimensional oriented subspace. We define in $O_{n+1}$ the set of $n$ circles $\left\{c_{1}(\tilde{f}), \ldots, c_{n}(\tilde{f})\right\}$ passing through $\tilde{f}$ and given by the relation

$$
c_{i}(\tilde{f})=\left\{\mathbf{l}_{1} \subset \cdots \subset \mathbf{l}_{i-1} \subset L_{i} \subset \mathbf{l}_{i+1} \subset \cdots \subset \mathbf{l}_{n+1}\right\} \quad i=1, \ldots, n
$$

where $L_{i}$ runs over the set of all $i$-dimensional subspaces satisfying the above inclusions and having the appropriate orientation, i.e. such that $\tilde{f}$ itself belongs to $c_{i}(\tilde{f})$.

The tangent lines to $c_{1}(\tilde{f}), \ldots, c_{n}(\tilde{f})$ at $\tilde{f}$ are linearly independent. They also have prescribed orientations meaning that the orientation of $\mathbf{l}_{i}$ appended by the velocity vector of the rotation of $L_{i}$ around $\mathbf{l}_{i-1}$ gives the orientation of $\mathbf{l}_{i+1}$. Let $c_{i}^{+}(\tilde{f})$ denote the chosen tangent halfline to $c_{i}(\tilde{f})$. Take the open $n$-dimensional orthant $C_{\tilde{f}} \in T O_{n+1}$ spanned by all $c_{i}^{+}(\tilde{f}), i=1, \ldots, n$. The distribution $\mathfrak{C}=\bigcup_{\tilde{f} \in O_{n+1}} C_{\tilde{f}}$ is called the Cartan distribution on the space $O_{n+1}$. In Lie-theoretic terms this distribution can be described as follows. $\mathfrak{C}$ coincides with the left-invariant distribution on the group $O_{n+1}$ which is generated by the cone of skew-symmetric matrices having all vanishing entries except those on the 1st upperdiagonal which are positive and, respectively, those on the 1st lowerdiagonal which are negative.

3.2.1. LEMmA, SEE [BSh]. The flag lift of any locally convex curve to $O_{n+1}$ is tangent to $\mathfrak{C}$.

It is fairly easy to see that the image of this Cartan distribution restricted to the group $T$ (considered as the open cell in $O_{n+1}$ ) coincides with the left-invariant distribution generated by the cone of all positive linear combinations of the entries on the 1st upperdiagonal in the Lie algebra $\mathfrak{T}$ of $T$ consisting of all nilpotent uppertriangular matrices. We call this distribution the Cartan distribution on $T$ and by abuse of notation we denote it by $\mathfrak{C}$ as well. The following result is classical and crucial in understanding of Theorem 3.2. 
3.2.2. Loewner-Whitney theorem, see e.g. Th. 1.1. in [FZ]. The Cartan distribution $\mathfrak{C}$ on $T$ has the property that the accessibility domain of any matrix $m \in T$ (i.e. the set of all end points of the curves starting at $m$ and tangent to $\mathfrak{C}$ ) coincides with $m \circ T^{+}$.

The idea of the proof is as follows. Let us fix any irreducible decomposition $\bar{i}=$ $\left(i_{1}, \ldots, i_{\left(\begin{array}{c}n \\ 2\end{array}\right)}\right)$ of the longest permutation $(n, n-1, n-2, \ldots, 1)$. Define the map $\Psi_{\bar{i}}$ : $\left(\mathbb{R}^{+}\right)\left(\begin{array}{c}n \\ 2\end{array}\right) \rightarrow T$ which sends a $\left(\begin{array}{l}n \\ 2\end{array}\right)$-tuple of positive numbers $\left(\tau_{1}, \ldots, \tau_{\left(\begin{array}{c}n \\ 2\end{array}\right)}\right)$ to the product of $T_{\tau_{1}, i_{1}} \circ T_{\tau_{1}, i_{1}} \circ \ldots \circ T_{\tau_{\left(\begin{array}{c}n \\ 2\end{array}\right)}, i\left(\begin{array}{c}n \\ 2\end{array}\right)}$ where $T_{\tau_{k}, i_{k}}$ is the uppertriangular unipotent matrix whose only nonzero element except the main diagonal equals $\tau_{k}$ and stands at the $i_{k}$-th row and $i_{k+1}$-st column. One can show that the image of $\Psi_{\bar{i}}$ coincides with $T^{+}$with some subset of real codimension at least 2 removed. Thus the closure of the image equals the set of all totally nonnegative matrices. This guarantees that the accessibility domain of the identity matrix is the whole $T^{+}$which together with the left-invariance of our distribution implies the required result.

This statement together with Theorem 3.1 and the interpretation of $T$ as an open Schubert cell in $O_{n+1}$ prove Theorem 3.2.

The next two simple lemmas can be found in e.g. [VD].

3.3. Lemma. For any triple of pairwise nonintersecting lines $l_{1}, l_{2}, l_{3}$ in $\mathbb{R} P^{3}$ there exists a unique hyperboloid $H$ of one sheet containing $l_{1}, l_{2}, l_{3}$.

Take any 4 -tuple $L$ of pairwise nonintersecting lines $L=\left(l_{1}, l_{2}, l_{3}, l_{4}\right)$ in $\mathbb{R} P^{3}$.

3.4. Lemma. There exist two real lines intersecting each line in $L$ if and only if $l_{4}$ intersects in two distinct points the hyperboloid $H$ containing $l_{1}, l_{2}, l_{3}$. (This property is independent on the particular choice of a triple in $L$. )

3.5. Main EXAmple. The following 4-tuple of lines in $\mathbb{R} P^{3}$ (given in homogeneous coordinates in $\mathbb{R}^{4}$ ) satisfies the conditions of Theorem 3.2 and settles Theorem B.

$$
\begin{gathered}
l_{1}=\left(\begin{array}{llll}
1 & 0 & 0 & 0 \\
0 & 1 & 0 & 0
\end{array}\right), \quad l_{2}=\left(\begin{array}{cccc}
1 & 1 & 1 / 2 & 1 / 6 \\
0 & 1 & 1 & 1 / 2
\end{array}\right), \\
l_{3}=\left(\begin{array}{cccc}
1 & 101 / 100 & 401 / 200 & 2503 / 1500 \\
0 & 1 & 5 / 2 & 5 / 2
\end{array}\right), \quad l_{4}=\left(\begin{array}{llll}
0 & 0 & 1 & 0 \\
0 & 0 & 0 & 1
\end{array}\right) .
\end{gathered}
$$

The totally positive uppertriangular matrices $M_{i, i+1}$ sending $l_{i}$ to $l_{i+1}$ are as follows

$$
M_{1,2}=\left(\begin{array}{cccc}
1 & 1 & 1 / 2 & 1 / 6 \\
0 & 1 & 1 & 1 / 2 \\
0 & 0 & 1 & 1 \\
0 & 0 & 0 & 1
\end{array}\right), \quad M_{2,3}=\left(\begin{array}{cccc}
1 & 1 / 100 & 1 / 200 & 1 / 500 \\
0 & 1 & 3 / 2 & 1 \\
0 & 0 & 1 & 1 \\
0 & 0 & 0 & 1
\end{array}\right)
$$

Finally, $l_{4}=\lim _{t \rightarrow+\infty} l_{3} M_{3,4}(t)$, where

$$
M_{3,4}(t)=\left(\begin{array}{cccc}
1 & t & t^{2} / 2 & t^{3} / 6 \\
0 & 1 & t & t^{2} / 2 \\
0 & 0 & 1 & t \\
0 & 0 & 0 & 1
\end{array}\right)
$$


Proof. Verified by direct calculation.

3.6. Lemma. The line $l_{3}$ does not intersect the hyperboloid $H \subset \mathbb{R} P^{3}$ containing $l_{1}, l_{2}, l_{4}$.

Proof. Indeed, the hyperboloid $H$ is given by the equation $\Phi=2 x_{1} x_{3}-3 x_{1} x_{4}-3 x_{2} x_{3}+$ $6 x_{2} x_{4}$. If we apply to the line $l_{2}$ an uppertriangular matrix

$$
M=\left(\begin{array}{cccc}
1 & a & b & c \\
0 & 1 & d & e \\
0 & 0 & 1 & f \\
0 & 0 & 0 & 1
\end{array}\right)
$$

we get the line $M\left(l_{2}\right)$ given by $\left(\begin{array}{cccc}1 & a+1 & b+d+1 / 2 & c+e+f / 2+1 / 6 \\ 0 & 1 & d+1 & e+f+1 / 2\end{array}\right)$.

Take the affine line in $\mathbb{R}^{4}$

$$
l l=\left\{\begin{array}{l}
x_{1}=1 \\
x_{2}=1+a+u \\
x_{3}=1 / 2+b+d+u(d+1) \\
x_{4}=1 / 6+c+e+f / 2+u(1 / 2+f+e)
\end{array}\right.
$$

belonging to $l_{3}$ (considered as a 2 -dimensional subspace in $\mathbb{R}^{4}$ ). The restriction of the defining equation of $H$ to $l l$ (calculated using Mathematica) is given by

$$
\begin{gathered}
\Phi(l l)=u^{2}(-3 d+6 e+6 f)+u(-3 b+6 c-4 d-3 a d+9 e+6 a e+6 f+6 a f)- \\
a / 2-b-3 a b+3 c+6 a c-d-3 a d+3 e+6 a e+3 f / 2+3 a f .
\end{gathered}
$$

The discriminant of this quadratic in $u$ equation equals

$$
\begin{aligned}
D s c(l l)= & 9 b^{2}-36 b c+36 c^{2}-6 a d+12 b d-18 a b d-12 c d+36 a c d+4 d^{2}-12 a d^{2}+9 a^{2} d^{2}+12 a e- \\
& 30 b e+36 a b e+36 c e-72 a c e-12 d e+42 a d e-36 a^{2} d e+9 e^{2}-36 a e^{2}+36 a^{2} e^{2}+12 a f- \\
& 12 b f+36 a b f-72 a c f-6 d f+24 a d f-36 a^{2} d f-36 a e f+72 a^{2} e f+36 a^{2} f^{2} .
\end{aligned}
$$

Observe that if $a=b=c=0$ then $D s c_{l l}=4 d^{2}-12 d e+9 e^{2}-6 d f=(2 d-3 e)^{2}-6 d f$. If one additionally assumes that $d>0, e>0, f>0$ and $d f>e$ then the corresponding matrix $M$ is totally nonnegative. One can easily adjust the values of $d, e, f$ to get the negative value of $D s c(l l)$. For example, $d=3 / 2, e=f=1$ gives $D s c(l l)=-9$. Since the space of all totally nonnegative matrices is the closure of the space of all totally positive matrices (see $[\mathrm{Wh}]$ ) one can find a small deformation of the later matrix which is totally positive but $D s c(l l)$ is still negative. The matrix $M_{2,3}$ above is totally positive with $l_{3}=l_{2} M_{2,3}$ such that $D s c(l l)=-2231979 / 250000 \approx-8.92792$.

To complete the proof of Theorem B notice that by Theorem 3.2 there exists a convex curve whose tangent lines are $l_{1}, l_{2}, l_{3}, l_{4}$ and thus by lemma 3.4 there are no real projective lines intersecting all four of them. In fact, in order to get the exact set-up of Theorem 3.2 we need to take instead of the line $l_{4}$ the line $l_{3} M_{3,4}(t)$ for some sufficiently large $t$, see the above notation. 


\section{§4. Some Grassmann Geometry, etc.}

1ST CONJECTURE. Let as above $G_{k+1, n+1}$ denote the usual Grassmannian of ( $k+$ 1 )-dimensional real linear subspaces in $\mathbb{R}^{n+1}$ (or equivalently, $k$-dimensional projective subspaces in $\left.\mathbb{R} P^{n}\right)$.

Definition. Given an $(n-k)$-dimensional subspace $L \subset \mathbb{R}^{n+1}$ we define the Grassmann hyperplane $H_{L} \subset G_{k+1, n+1}$ associated to $L$ to be the set of all $(k+1)$-dimensional subspaces in $\mathbb{R}^{n+1}$ nontransversal to $L$.

Definition. A smooth closed curve $\gamma: S^{1} \rightarrow G_{k+1, n+1}$ is called locally Grassmann convex if the local multiplicity of intersection of $\Gamma$ with any Grassmann hyperplane $H_{L} \subset$ $G_{k+1, n+1}$ does not exceed $(k+1)(n-k)=\operatorname{dim} G_{k+1, n+1}$ and globally Grassmann convex or simply Grassmann convex if the above condition holds for the global multiplicity.

Given a locally convex curve $\gamma: S^{1} \rightarrow \mathbb{R} P^{n}$ and $1 \leq k \leq n-1$ we can define its $k$-th Grassmann lift $\gamma_{G}^{k}: S^{1} \rightarrow G_{k+1, n+1}$ formed by the osculating $k$-dimensional projective subspaces to the initial $\gamma$. (The curves $\gamma_{G}^{k}$ are well-defined for any $k=1, \ldots, n-1$ due to the local convexity of $\gamma$.)

The following is the reformulation of the first basic conjecture from the introduction.

Conjecture on the $k$-th developable restated. For any convex $\gamma: S^{1} \rightarrow \mathbb{R} P^{n}$ its $k$-th Grassmann lift $\gamma_{G}^{k}$ is Grassmann convex.

The scheme of the proof of Theorem A in $\S 2$ can be generalized to higher dimensions but the number of cases increases drastically. One apparently needs a different inductive argument.

2ND CONJECTURE. Recall that an enumerative problem in complex algebraic geometry is called totally real if all objects involved can be realized in the real subspace of the complex space. There is a large number of enumerative problems known to be totally real. On the other hand, there exists an impressive list of those which fail to be totally real, see [So3] and references therein.

The following question was raised by W. Fulton in $[\mathrm{F}]$.

Problem. Do there exist configurations of $(k+1)(n-k)$-tuples of $k$-dimensional subspaces in $\mathbb{R} P^{n} \subset \mathbb{C} P^{n}$ such that all $\sharp_{k, n}$ possible complex $(n-k-1)$-dimensional complex subspaces in $\mathbb{C} P^{n}$ intersecting each subspace in the $(k+1)(n-k)$-tuple are real? (Such configurations of subspaces are called totally real.)

Conjecture on total reality restated. For the rational normal curve $\rho_{n}: S^{1} \rightarrow \mathbb{R} P^{n}$ the configuration of any its $(k+1)(n-k)$ pairwise distinct projective $k$-dimensional osculating subspaces is totally real.

Identifying the space $\mathbb{R} P^{n}$ with the space of all polynomials in one variable of degree at most $n$ (considered up to a constant factor) and the rational normal curve with the family $(x-\alpha)^{n}$ one gets the reformulation of the conjecture on total reality in terms of rational curves given in $\S 1$. The most promising approach to this conjecture seems to be the study of the geometry of the Wronski map from Grassmannians to projective spaces, see e.g. [EG2]. Namely, consider the map $W_{k, n}: G_{k+1, n+1} \rightarrow \mathbb{R} P^{(k+1)(n-k)}$ associating to a $(k+1)$-dimensional linear subspace in the space of polynomials its Wronskian, i.e. 
the determinant of the $(k+1) \times(k+1)$ matrix

$$
\left(\begin{array}{cccc}
p_{1}(x) & p_{2}(x) & \ldots & p_{k+1}(x) \\
p_{1}^{\prime}(x) & p_{2}^{\prime}(x) & \ldots & p_{k+1}^{\prime}(x) \\
p_{1}^{\prime \prime}(x) & p_{2}^{\prime \prime}(x) & \ldots & p_{k+1}^{\prime \prime}(x) \\
\vdots & \vdots & \ddots & \vdots \\
p_{1}^{(k)}(x) & p_{2}^{(k)}(x) & \ldots & p_{k+1}^{(k)}(x)
\end{array}\right)
$$

where $p_{1}(x), \ldots, p_{k+1}(x)$ is some basis of the chosen subspace.

Note that any change of a basis of a given subspace results in multiplying the Wronskian by a constant. Wronskians are polynomials of degree at most $(k+1)(n-k)$. (The Wronski map is much more natural over $\mathbb{C}$ but we are doing real algebraic geometry at the moment.) One can now define two hypersurfaces in the image space $\mathbb{R} P^{(k+1)(n-k)}$ (identified with the space of polynomials of degree at most $(k+1)(n-k)$.) The first hypersurface $\mathcal{D} \subset$ $\mathbb{R} P^{(k+1)(n-k)}$ is the usual discriminant consisting of all polynomials with multiple zeros. The second hypersurface $\mathcal{J} \subset \mathbb{P}^{(k+1)(n-k)}$ is the image of the hypersurface in $G_{k+1, n+1}$ where the Jacobian of $W_{k, n}$ vanishes, i.e. where $W_{k, n}$ is not a local diffeomorphism. If one could settle the following conjecture then together with the main result of [So2] this would prove the conjecture on total reality.

$\mathcal{D}-\mathcal{J}$-conjecture. The hypersurface $\mathcal{J}$ does not intersect the domain of all strictly hyperbolic polynomials, i.e. polynomials whose zeros are all real and distinct.

Problem. Enumerate irreducible components of $\mathcal{D} \cap \mathcal{J}$.

On PROJECTIONS OF CONVEX CURVES. The following question seems to be of importance in computer graphics applications.

Problem. Given a real plane closed smooth curve $\gamma$ such that the supremum of the total number of its intersection points with affine lines is finite represent $\gamma$ as a projection of a convex curve lying in the projective space of some dimension.

At the moment it is not even proved (although very plausible) that such a representation always exists. Assuming that it exists one wants to determine the minimal dimension such that a given plane curve can be obtained projecting a convex curve lying in the projective space of this dimension. The obvious guess (which the authors encounted in the applied literature) that this dimension coincides with the above mentioned supremum of total number of intersections is definitely wrong. We finish the article with the following statement.

Conjecture. A closed real smooth curve $\gamma$ in $\mathbb{P}^{2}$ can be obtained as a projection of a convex curve in $\mathbb{P}^{3}$ if and only if the following holds:

i) the maximal total intersection of $\gamma$ with any line in $\mathbb{P}^{2}$ equals 3 ;

ii) the maximal number of inflection points of $\gamma$ equals 3 ;

iii) the maximal number of tangent lines to $\gamma$ through any point on $\mathbb{P}^{2}$ equals 4 .

\section{REFERENCES}

[A1] V. I. Arnold, On the number of flattening points on space curves, Sinai's Moscow seminar on Dynamical Systems, AMS Trans., Ser. 2, vol. 171, 1995.

[A2] V. I. Arnold, Towards the Legendrian Sturm theory of space curves, Funct. Anal. Appl (1998), no. $2,1-7$. 
[BSh] B. Shapiro, Space of linear differential equations and flag manifolds, Math. USSR - Izv. 36 (1991), no. 1, 183-197.

[EG1] A. Eremenko, A. Gabrielov, Rational functions with real critical points and B. and M. Shapiro conjecture in real enumerative geometry, Annals of Math 155 (2002), no. 1, 105-129.

[EG2] A. Eremenko, A. Gabrielov, Degrees of real Wronski map, Discrete Comput. Geom 28 (2002), no. 3, 331-347.

[F] W. Fulton, Introduction to intersection theory in algebraic geometry, CBMS 54, AMS, 1984.

[FZ] S. Fomin and A. Zelevinsky, Totally nonnegative and oscillatory elements in semisimple groups, Proc. Amer. Math. Soc 128 (2000), 3749-3759.

[Ga] F. Gantmakher, The theory of matrices. Vol. 1, AMS Chelsea Publishing, Providence, RI, 1998, pp. $\mathrm{x}+374$, Reprint of the 1959 translation.

[KS] S. Karlin and W. Stadden, Tchebycheff systems: With applications in analysis and statistics, Pure and Applied Mathematics XV (1966), Interscience Publishers John Wiley \& Sons, 586 pp.

[MSh] M. Shapiro, The topology of the space of nondegenerate curves, Russian Acad. Sci. Izv. Math 43 (1994), no. 2, 291-310.

[Po] G. Polya, On the mean value theorem corresponding to a given linear ordinary differential equations, Trans. AMS 24 (1924), no. 4, 312-324.

[RSSS] J. Ruffo, Y. Sivan, E. Soprunova, and F. Sottile, Experimentation and conjectures in the real Schubert calculus for flag manifolds, math.AG/0502040.

[Sch] H. Schubert, Beziehungen zwischen den linearen Räumen auferlegbaren charakteristischen Bedingungen, Math. Ann 38 (1891), 598-602.

[Se] V. Sedykh, Some invariants of admissible homotopies of space curves, Funct. Anal. Appl 35 (2001), no. 4, 284-293.

[So1] F. Sottile, Real Schubert calculus: polynomial systems and a conjecture of Shapiro and Shapiro, Experimental Math 9 (2000), no. 2, 161-182.

[So2] F. Sottile, The special Schubert calculus is real, Electronic Res. Ann. of the AMS 5 (1999), 35-39.

[So3] F. Sottile, Some real and unreal problems in enumerative geometry of flag manifolds, in special volume dedicated to William Fulton on the occasion of his 60th birthday, Michigan Math. J 48 (2000), 573-592.

[So4] F. Sottile, Shapiro and Shapiro conjecture, website - www.expmath.org/extra/9.2/sottile.

[SS] B. Shapiro, M. Shapiro, Projective convexity in $\mathbb{P}^{3}$ implies Grassmann convexity, Int. J. Math 11 (2000), no. 4, 579-588.

[To] B. Totaro, Space curves with nonzero torsion, Int. J. Math 1 (1990), no. 1, 109-117.

[VD] O. Viro and Yu. Drobotukhina, Configurations of skew-lines, Leningrad Math. J 1 (1990), no. 4, 1027-1050.

[Wh] A. Whitney, A reduction theorem for totally positive matrices, J. d'Analyse Math 2 (1952), 88-92. 